CAPÍTULO 6

\title{
O USO DA ESTATÍSTICA SCAN PARA DETECÇÃO DE AGLOMERADOS DOS ALERTAS DE DESMATAMENTOS NA APA TRIUNFO DO XINGU, PA - BRASIL
}

\author{
DOI: http://dx.doi.org/10.18616/planar06
}

Ivan Merêncio

Carlos Antônio Oliveira Vieira 


\section{INTRODUÇÃO}

As unidades de conservação (UC) são espaços territoriais, com normas e regras especiais, que visam assegurar a representatividade ecológica das diferentes espécies, habitats e ecossistemas do território nacional, preservando o patrimônio biológico existente. Dentre os diversos tipos de unidades, existe a área de proteção ambiental (APA), que é caracterizada como uma unidade de uso sustentável (BRASIL, 2000).

$\mathrm{O}$ art. 15 da lei federal n. 9.985 de 2000 define a APA como uma área em geral extensa, com um certo grau de ocupação humana, dotada de atributos abióticos e bióticos, que tem como objetivos básicos proteger a diversidade biológica, orientar o processo de ocupação e garantir a sustentabilidade do uso dos recursos naturais (BRASIL, 2000). Entretanto, Souza Júnior et al. (2018) explica que as áreas protegidas (APs) da Amazônia estão ameaçadas e sofrem intensa pressão antrópica, que é caracterizada como o desmatamento no entorno das áreas protegidas num raio de $10 \mathrm{~km}$. Em 2016 ocorreu devastação florestal de $1.225 \mathrm{~km}^{2}$ dessas APs, o que representou $15,5 \%$ do total da supressão florestal na Amazônia naquele ano.

A APA Triunfo do Xingu, instituída em 2006 pelo governo estadual do Pará, é uma unidade de conservação que está sofrendo com a iminente expansão do desmatamento em seu interior. Entre 2012 e 2014 houve a supressão de 27.700ha de sua vegetação, o que a classificou como a UC brasileira em que ocorreu mais desmatamento para o período (ARAÚJO et al., 2017). E também como a área protegida mais pressionada entre 2016 e 2017 (SOUZA JÚNIOR et al., 2018). Nessa circunstância, é necessário a adoção de métodos e tecnologias que sejam capazes de aumentar a eficiência das medidas de controle. A estatística scan (ou de varredura), originalmente apresentada por Kulldorff et al. (2005), é uma ferramenta capaz de cumprir essa função. Ela é utilizada para a avaliação estatística do objeto de estudo no espaço geográfico, com a finalidade de detectar e hierarquizar aglomerados em regiões que demandam maior atenção (ARAÚJO, 2012). 
A estatística scan busca detectar uma região composta por aglomerados, comparando o número de casos observados em relação aos esperados. Esses agrupamentos são constituídos através da modificação do raio, que varia de zero até um valor máximo de $50 \%$ da população em risco (neste caso a floresta) no interior do cilindro (RANGEL, 2016). Determina-se a razão entre a verossimilhança com a hipótese alternativa de que o risco de acontecer o evento é maior dentro do que fora do aglomerado. A hipótese nula é de que a divergência entre as duas verossimilhanças ocorra ao acaso (KAQUI, 2016).

Diante da intensa pressão antrópica em que essa unidade de conservação está sendo submetida, este artigo tem como objetivo detectar e hierarquizar os aglomerados dos alertas de desmatamentos na APA Triunfo do Xingu ao nível de significância de 5\%, classificando-os conforme a prioridade de intervenção.

\section{MATERIAIS E MÉTODOS LOCALIZAÇÃO DA ÁREA DE ESTUDO}

A APA Triunfo do Xingu está localizada na Região do Norte do Brasil, no estado do Pará. Situada entre as latitudes 05०36’00" e 070 15’ 02"

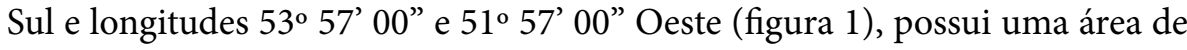
$16.792,81 \mathrm{~km}^{2}$ (SOUZA JÚNIOR, 2018).

Essa APA foi instituída em 2006 através do decreto estadual n. 2.612 com o objetivo de proteger a diversidade biológica e assegurar a sustentabilidade do uso dos recursos naturais, para a melhoria da qualidade de vida da população local. Cerca de $66 \%$ de sua área está estabelecida no munícipio de São Félix do Xingu e 34\% em Altamira (PARÁ, 2006).

A área protegida pertence ao bioma Amazônico e possui as fitofisionomias de Floresta Ombrófila Aberta (53,20\%) Floresta Ombrófila Densa $(39,61 \%)$ e Savana-Floresta Ombrófila $(7,19 \%)$ (ISA, 2012). No verão, o rio Xingu proporciona áreas de lazer nas ilhas, sendo ponto turístico para pessoas 
de diversas regiões do estado, possibilitando inclusive a prática da pesca esportiva (IDEFLOR-BIO, 2015).

Figura 1 - Mapa de localização da área de estudo

MAPA DE LOCALIZAÇÃO DA APA TRIUNFO DO XINGU - PA - BRASIL
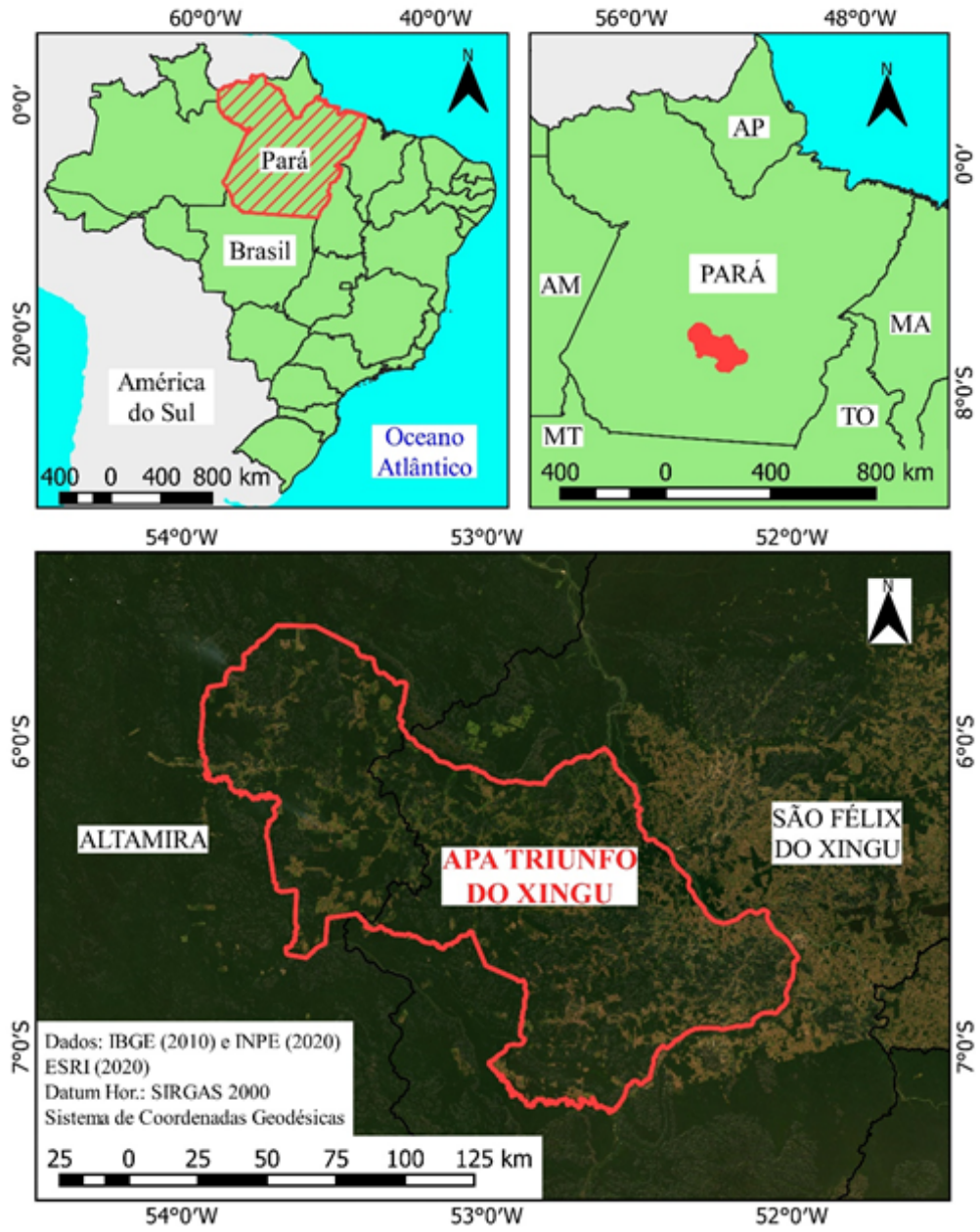

Fonte: Autores (2020). 


\section{MATERIAIS}

Para o desenvolvimento da análise espaço-temporal utilizou-se de pesquisa bibliográfica e documental. Os alertas diários de desmatamento da Amazônia legal do período de 2017 a 2019, produzidos pelo Sistema de Detecção do Desmatamento na Amazônia Legal em Tempo Real (DETER), foram obtidos no Portal TerraBrasilis do Instituto Nacional de Pesquisas Espaciais (INPE). O arquivo de acesso público, estava no formato shapefile, Datum SIRGAS 2000, e continha os polígonos das áreas em que houve degradação florestal no intervalo de estudo. Os limites das APAs da Amazônia Legal também foram obtidos pelo Portal TerraBrasilis.

A base vetorial contendo os limites municipais e do estado do Pará do ano de 2010 foi disponibilizada pelo IBGE em seu portal, em extensão shapefile, Datum SIRGAS 2000 e no Sistemas de Coordenadas Geodésicas.

\section{MÉTODO}

As fases de execução da detecção dos aglomerados, contemplando a sistematização dos dados, a escolha do modelo de probabilidade, a definição dos parâmetros estatísticos e a aplicação da estatística scan estão resumidas no fluxograma da figura 2 e são descritos a seguir.

Figura 2 - Fluxograma de execução do método

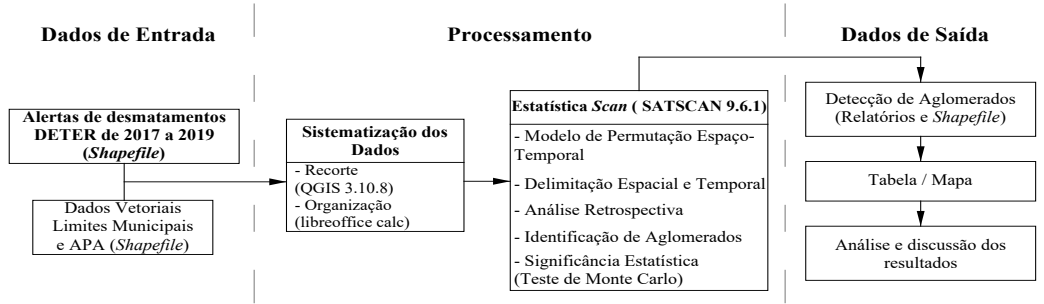

Fonte: Autores (2020). 
A sistematização da base de dados iniciou no programa QGIS 3.10.8. Extraiu-se apenas os polígonos dos alertas contidos na área de interesse. Em seguida, determinou-se os pontos centrais dos desflorestamentos no QGIS 3.10.8. Na etapa seguinte foi definido as coordenadas geodésicas decimais desses pontos.

O programa LibreOffice Calc foi aplicado para a organização da base descritiva. Definiu-se um número inteiro aleatório para identificação dos alertas, e incluiu-se a quantidade de ocorrências de devastação florestal em cada ponto. Desse modo, obteve-se os atributos essenciais para a execução da estatística: identificador do alerta (gerado pelos autores), número de casos de desmatamento, ano de detecção, e suas respectivas coordenadas geodésicas decimais.

\section{ESTATÍSTICA SCAN: MODELO PERMUTAÇÃO ESPAÇO TEMPORAL}

Para verificar a existência dos aglomerados utilizou-se o software SATSCAN 9.6.1, apoiado no modelo de permutação espaço-temporal (KULLDORFF et al., 2005), pois os registros de desflorestamentos são dados oriundos de contagem, e não era possível a quantificação da população em risco, que nesse caso é a floresta. O uso dos modelos exponencial, normal e ordinal não era adequado ao objetivo da pesquisa. Nesse sentido, a variável resposta foi o número de registros de desmatamentos detectados na APA Triunfo do Xingu entre 2017 e 2019, que são dados pontuais com centroides localizados no interior da área de proteção.

A estatística scan atua com a varredura de diversos raios de busca, por isso foi necessário definir esse limite. Assim, estipulou-se como parâmetro máximo de detecção espacial o valor de $20 \mathrm{~km}$, fundamentado no estudo de Balieiro (2008), que aplicou a estatística scan em ocorrências de supressão florestal em alguns municípios do sul do estado do Amazonas, para garantir que a fiscalização do aglomerado detectado fosse viável espacialmente. 
Devido a escolha pelo modelo de permutação espaço-temporal foi necessário informar o limite de busca no tempo. Desse modo, definiu-se como indicador temporal máximo o intervalo de 1 ano, atendendo ao objetivo desse estudo.

A busca dos aglomerados foi baseada na análise retrospectiva, visando detectar tanto os agrupamentos que deixaram de existir quanto os que permaneceram ativos até o fim do intervalo do estudo. Para cada suposto aglomerado foi calculado o valor da Razão de Verossimilhança Generalizada de Poisson (RVG), aquele que apresentasse o maior valor, era o mais provável, denominado de primário. Para o teste da significância desse conglomerado primário considerou-se $5 \%$ de probabilidade, com as seguintes hipóteses:

$\mathrm{H}_{0}$ : não existe aglomerado espaço-temporal dos alertas de desmatamento na APA Triunfo do Xingu;

$\mathrm{H}_{1}$ : existe aglomerado espaço-temporal dos alertas de desmatamento na APA Triunfo do Xingu.

O procedimento de Monte Carlo foi empregado para testar essas hipóteses com relação ao aglomerado primário. E após as 999 permutações se a ordem $\mathrm{R}$ estivesse acima de 950, contatava-se que o agrupamento das ocorrências naquele aglomerado não aconteceu devido ao acaso. Os conglomerados com valores de RVG menores, dito secundários, tiveram avaliação da significância de modo similar ao realizado no primário.

Por fim, utilizou-se o software QGIS 3.10.8 para espacialização dos resultados obtidos no SATSCAN 9.6.1, finalizando-os com a criação de tabelas e do mapa de aglomerados dos alertas de desmatamentos detectados na Área de Proteção Ambiental Triunfo do Xingu entre 2017 e 2019.

\section{RESULTADOS E DISCUSSÃO}

O DETER registrou 3.244 alertas de desmatamentos na APA Triunfo do Xingu no período dos anos de 2017 a 2019. Dos quais, 65,57\% ocorreram 
no município de São Félix do Xingu e 34,33\% em Altamira. Inclusive, nesses três anos, os registros sempre foram mais elevados no munícipio de São Félix do Xingu, com crescimento intenso em 2019 (gráfico 1).

Gráfico 1 - Alertas de desmatamentos da APA Triunfo do Xingu distribuídos por município.

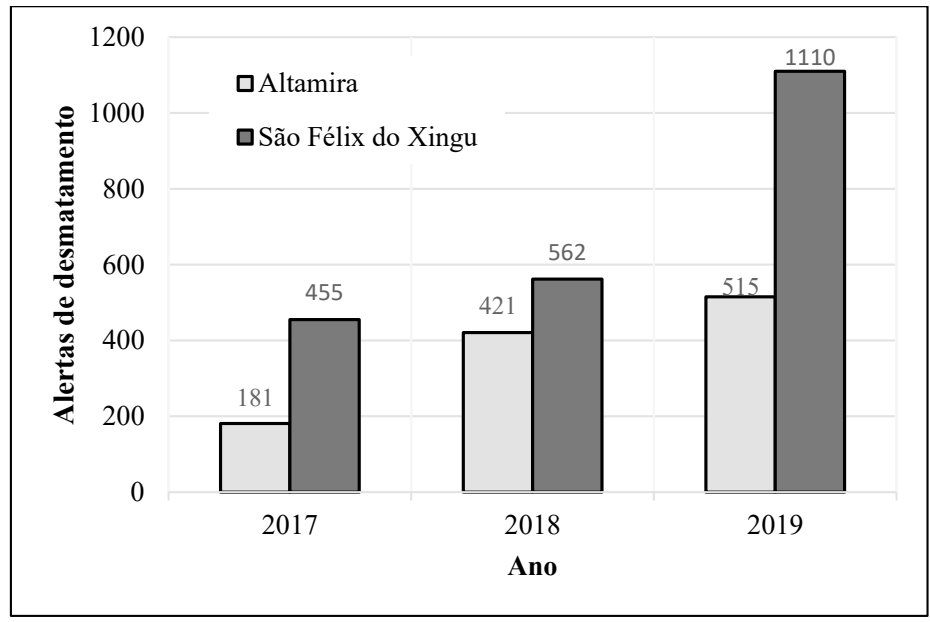

Fonte: Autores (2020).

Os números estimados apontam que foram degradados $1.731,98$ $\mathrm{km}^{2}$ de florestas em São Félix do Xingu e $826,68 \mathrm{~km}^{2}$ em Altamira, totalizando $2.558,66 \mathrm{~km}^{2}$ de desmatamentos em toda APA, porém salienta-se que os dados de área servem apenas como estimativas, para obtenção de valores precisos é necessário consultar os dados do Projeto de Monitoramento do Desmatamento na Amazônia Legal por Satélite (PRODES).

A estatística scan identificou doze aglomerados significativos ( $\mathrm{p}<0,05)$ entre os anos de 2017 e 2019 (tabela 1). O aglomerado primário (P1) do ano 2017 teve centroide em São Félix do Xingu, como mostra a figura 3 (A), com uma RVG de 28,96 e um raio de 19,79 km. Nessa área, no prazo de um ano, ocorreram 115 registros de desmatamento. Sob a hipótese nula, de 
que não existisse agrupamento, seriam esperados 52 casos. O p-valor para este aglomerado, obtido a partir de 999 simulações pelo método de Monte Carlo, foi igual a $5,22 \times 10^{-15}$. Como o valor resultante é menor que 0,05 a hipótese nula, da não existência do aglomerado, foi rejeitada, o que indica que o valor foi significativo. Os outros agrupamentos foram analisados de modo análogo ao primário de 2017. Os aglomerados primários (P1) de 2018 e 2019 ficaram situados no munícipio de Altamira.

Tabela 1 - Aglomerados dos alertas de desmatamentos detectados na Área de Proteção Ambiental Triunfo do Xingu pela estatística scan entre 2017 e 2019.

\begin{tabular}{|c|c|c|c|c|c|c|c|c|}
\hline ID & $\begin{array}{l}\text { Prioridade } \\
\text { anual }\end{array}$ & Localização & $\begin{array}{l}\text { Raio } \\
(\mathbf{k m})\end{array}$ & Ano & RVG & p-valor & $\begin{array}{l}\text { Alertas } \\
\text { Obs. }\end{array}$ & $\begin{array}{l}\text { Alertas } \\
\text { Esp. }\end{array}$ \\
\hline 1 & $\mathrm{P} 1$ & São Félix do Xingu & 19,79 & 2017 & 28,96 & $5,22 \times 10^{-15}$ & 115 & 52 \\
\hline $\begin{array}{l}2 \\
\text { P1 }\end{array}$ & & Altamira & 19,99 & 2018 & 27,78 & $2,62 \times 10^{-14}$ & 165 & 88 \\
\hline 3 & $\mathrm{P} 1$ & Altamira & 8,83 & 2019 & 22,15 & $5,85 \times 10^{-11}$ & 120 & 62 \\
\hline 4 & S2 & São Félix do Xingu & 8,22 & 2018 & 17,91 & $1,93 \times 10^{-08}$ & 59 & 24 \\
\hline 5 & $\mathrm{~S} 2$ & Altamira & 8,90 & 2017 & 17,14 & $5,52 \times 10^{-08}$ & 28 & 7 \\
\hline 6 & S3 & São Félix do Xingu & 13,12 & 2017 & 14,50 & $2,05 \times 10^{-06}$ & 21 & 5 \\
\hline 7 & S3 & Altamira & 6,04 & 2018 & 13,97 & $4,19 \times 10^{-06}$ & 28 & 8 \\
\hline 8 & S4 & São Félix do Xingu & 12,12 & 2018 & 13,65 & $6,51 \times 10^{-06}$ & 90 & 49 \\
\hline 9 & $\mathrm{~S} 2$ & São Félix do Xingu & 6,76 & 2019 & 8,94 & $4,12 \times 10^{-03}$ & 51 & 27 \\
\hline 10 & S4 & São Félix do Xingu & 5,42 & 2017 & 8,63 & $6,23 \times 10^{-03}$ & 13 & 3 \\
\hline 11 & S5 & São Félix do Xingu & 8,85 & 2017 & 8,28 & $1,00 \times 10^{-02}$ & 20 & 7 \\
\hline 12 & S3 & São Félix do Xingu & 10,42 & 2019 & 7,38 & $3,60 \times 10^{-02}$ & 58 & 34 \\
\hline
\end{tabular}

Fonte: Autores (2020).

A Estatística scan identificou cinco agrupamentos em 2017 (figura 3 [A]), quatro em 2018 (figura 3 [B]) e três em 2019 (figura 3 [C]). Sendo que do número total, oito aglomerados concentraram-se em São Félix do Xingu e quatro em Altamira. Além disso, houve alteração do agrupamento mais verossímil no decorrer dos anos. 
Observou-se que a degradação das florestas esteve distribuída em toda região da APA, apresentando variação ao longo do tempo devido a expansão do desmatamento. Ainda assim, houve a predominância na área de abrangência de São Félix do Xingu, sendo que essa prevalência também foi constatada por Doblas (2015).

A distância de polos madeireiros é uma variável espacial que influencia no aumento das ocorrências de extração florestal (MATRICARDI, 2007; COSTA, 2017). Estima-se que essa variável seja a principal contribuinte do desmatamento na APA Triunfo do Xingu, pois o polo madeireiro mais próximo está situado na área urbana de São Félix do Xingu. Araújo et al. (2017) complementa que a ocupação irregular de terras e a ausência de demarcação das áreas de proteção são causas relevantes da supressão vegetal nessa UC.

Figura 3 - Mapa de aglomerados dos alertas de desmatamentos detectados na Área de Proteção Ambiental Triunfo do Xingu, situada no Pará, em 2017 (A),

$$
2018 \text { (B) e } 2019 \text { (C) }
$$

Mapa de aglomerados dos alertas de desmatamentos detectados na Área de Proteção Ambiental Triunfo do Xingu entre 2017 a 2019.
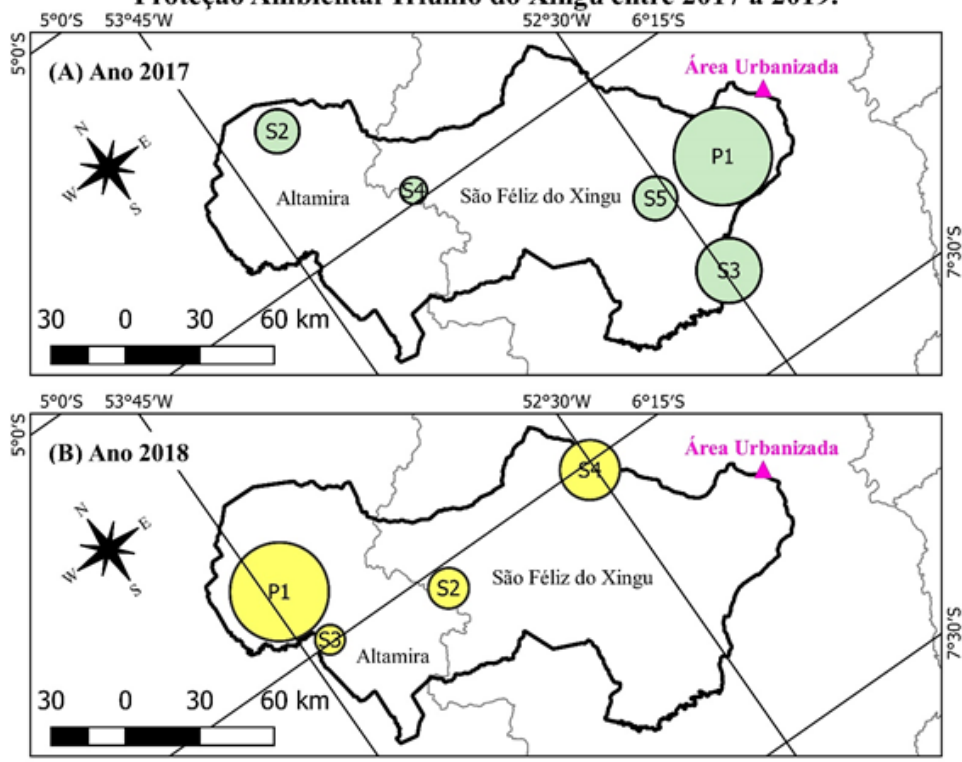


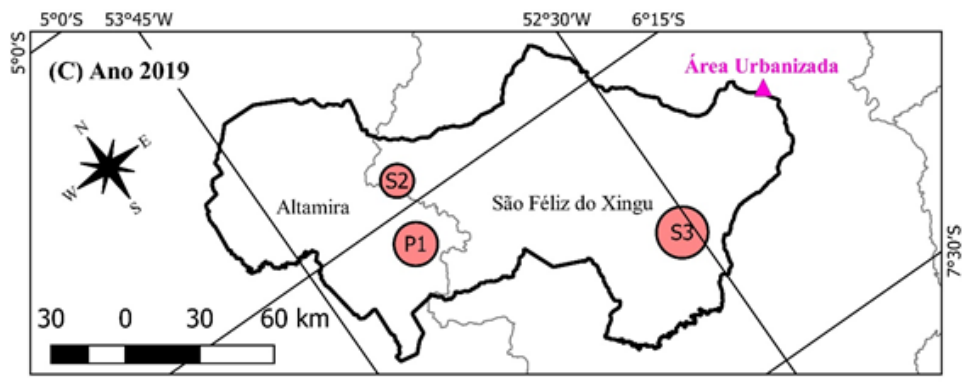

\begin{tabular}{|lll|}
\hline & \multicolumn{2}{c|}{ Legenda } \\
Dados: Deter - INPE (2020) & Área Urbana São Félix do Xingu \\
Base Cartográfica: IBGE (2010) & Laio do Cluster \\
Escala: 1 / 2.250.000 & Limites Municipais \\
Datum Hor.: SIRGAS 2000 & Ano 2017 \\
Sistema de Coordenadas Geodésicas & & \\
\hline
\end{tabular}

Fonte: Autores (2020).

Sabe-se ainda que 74\% da rede de estradas (não oficiais) dessa APA pertence ao município de São Félix do Xingu e o restante a Altamira (PINTO et al., 2011). Nepstad et al. (2001) e Silvestrini et al. (2011) associaram a proximidade com estradas ao aumento da degradação florestal, pois o custo de transporte da madeira é maior em locais mais distantes.

Castro et al. (2010) afirmam que a expansão da bovinocultura extensiva tem relação com altas taxas de desmatamentos, principalmente em regiões do Oeste do Maranhão, Sul e Sudeste do Pará, Norte do Mato Grosso, Rondônia e Acre. Nessa perspectiva, verificou-se que o munícipio de São Félix do Xingu teve o maior rebanho bovino do país em 2019, com efetivo de 2.241.537 animais (IBGE, 2019).

Costa (2017) afirma que a determinação de áreas suscetíveis a degradação é fundamental para frear processos de desmatamentos futuros. A estatística scan enquadra-se a essa premissa, pois possibilita identificar e quantificar agrupamentos de desmatamentos prioritários de intervenção (PINHEIRO et al., 2009). 


\section{CONSIDERAÇÕES FINAIS}

A detecção de aglomerados de ocorrências de desmatamentos na APA Triunfo do Xingu entre 2017 e 2019 evidenciou que a degradação florestal estava distribuída em toda sua área de abrangência, porém com predominância na porção territorial de São Félix do Xingu, cujo município conteve $66,67 \%$ dos aglomerados identificados, aproximadamente.

Nesse cenário, este artigo atingiu o objetivo de aplicar a estatística scan para a identificação dos agrupamentos dos alertas de desmatamento na APA Triunfo do Xingu ao nível de significância de 5\%, classificando-os conforme prioridade de intervenção.

Desse modo, a detecção e hierarquização de agrupamentos espaço-temporais significativos, com a representação dos resultados através de documentos cartográficos, demonstrou-se relevante para a identificação de áreas prioritárias, ou seja, é um procedimento de apoio ao planejamento e a execução de medidas para a contenção do desmatamento ilegal.

Esse tipo de pesquisa é de extrema importância em estudos direcionados a minimizar os impactos dos desmatamentos de unidades de conservação. Cabe aos órgãos ambientais responsáveis utilizarem desta ferramenta para o planejamento, gestão e fiscalização das áreas de proteção ambiental. A fiscalização de aglomerados prioritários torna-se mais vantajosa devido a extensa área da APA e o número de equipes disponíveis para essa tarefa.

Em trabalhos futuros sugere-se a inclusão de covariáveis no modelo para averiguar sua influência nos agrupamentos. Um fator pertinente que atenderia a essa finalidade seria a distância das estradas a esses alertas de desmatamentos. 


\section{REFERÊNCIAS}

ARAÚJO, E.; BARRETO, P.; BAIMA, S.; GOMES, M. Unidades de conservação mais desmatadas da Amazônia Legal (2012-2015). Belém: IMAZON, 2017. Disponível em: <http://imazon.org.br/PDFimazon/Portugues/livros/ UCS\%20mais\%20desmatadas\%20Amazonia_2012-2015.pdf >. Acesso em: 12 out. 2020.

ARAÚJO, T. C. Extensão da estatística Scan para detecção de conglomerados espaço-temporais em dados com excesso de zeros. 2012. 89 p. Dissertação (mestrado em Estatística), UnB, Brasília, 2012. Disponível em: <http://repositorio.unb.br/handle/10482/12804>. Acesso em: 10 out. 2020.

BALIEIRO, A. A. S. Detecção de conglomerados dos alertas de desmatamentos no Estado do Amazonas usando estatística de varredura espaço-temporal. 2008. 87 p. Dissertação (Mestrado em Estatística Aplicada e Biometria), Universidade Federal de Viçosa, Viçosa-MG, 2008.

BRASIL. Lei n. 9.985, de 18 de julho de 2000. Regulamenta o art. 225, $₫ 10$, incisos I, II, III e VII da Constituição Federal, institui o Sistema Nacional de Unidades de Conservação da Natureza e dá outras providências. Disponível em: <http://www.planalto.gov.br/ccivil_03/leis/19985.htm>. Acesso em: 14 out. 2020 .

CASTRO, E. et al. Atividade da pecuária, atores, mercado e cadeias produtivas. In: MENEZES, C. R. C. et al. (org.). Zoneamento Ecológico-Económico das Zonas Leste e Calha Norte do Estado do Pará. Belém: Núcleo de Gerenciamento do Programa Pará Rural, 2010. p. 123-153. 3v

COSTA, B. O. Degradação Florestal por extração seletiva e fogo na Amazônia Legal. 2017. 186 p. Tese (Doutorado em Ciências Florestais), Universidade de Brasília, Brasília-DF, 2017.

DOBLAS, J. Rotas do saque: violações e ameaças à integridade territorial da Terra do Meio (PA). São Paulo: Instituto socioambiental, 2015. 
INSTITUTO BRASILEIRO DE GEOGRAFIA E ESTATÍSTICA-IBGE. SIDRA: Pesquisa da Pecuária Municipal 2019. Disponível em: < https://sidra. ibge.gov.br/tabela/3939\#resultado>. Acesso em: 16 out. 2020.

INSTITUTO DE DESENVOLVIMENTO FLORESTAL E DA BIODIVERSIDADE DO ESTADO DO PARÁ - IDEFLOR-BIO. APA Triunfo do Xingu. Disponível em: <https://ideflorbio.pa.gov.br/unidades-de-conservacao/regiao-administrativa-do-xingu/apa -triunfo-do-xingu/>. Acesso em: 16 out. 2020 .

INSTITUTO SOCIOAMBIENTAL-ISA. De olho na Bacia do Xingu. São Paulo: ISA, 2012.

KAQUI, R. L. Uma Estatística de Varredura Espacial para Dados de Contagem com Censura. 2016. 98 f. Dissertação (mestrado em Estatística), UnB, Brasília, 2016. Disponível em: <https://repositorio.unb.br/handle/10482/23067>. Acesso em: 17 out. 2020.

KULLDORFF, M.; HEFFERNAN, R.; HARTMAN, J.; ASSUNÇÃO, R.; MOSTASHARI, F. A Space-Time Permutation Scan Statistic for Disease Outbreak Detection. Plos Medicine, v. 2, n. 3, p. 216-224, fev. 2005.

MATRICARDI, E. A. T. Spatiotemporal Dynamics of Forest degradation by selective logging and forest fire in the Brazilian Amazon. 2007. 270p. Departament of Geography, University of Michigan, EUA, 2007.

NEPSTAD, D.; CARVALHO, G.; BARROS, A. C.; ALENCAR, A.; CAPOBIANCO, J. P.; BISHOP, J.; MOUTINHO, P.; LEFEBVRE, P.; LOPES SILVA, U.; PRINS, E. Road paving, fire regime feedbacks, and the future of Amazon forests. Forest Ecology And Management, v. 154, n. 3, p. 395-407, dez. 2001.

PARÁ. Decreto estadual n. 2.612, de 4 de dezembro de 2006. Dispõe sobre a Criação da Área de Proteção Ambiental Triunfo do Xingu nos Municípios de São Félix do Xingu e Altamira, Estado do Pará, e dá outras providências. 
Disponível em: <https://www.semas.pa. gov.br/legislacao/files/pdf/546.pdf > Acesso em: 5 out. 2020.

PINHEIRO, J. G.; VIEIRA, C. A. O.; SANTOS, N. T.; BALIEIRO, A. A. da S. O uso do sensoriamento remoto e da estatística de varredura (scan) na detecção e quantificação em significância de agrupamentos de desmatamento no sul da Amazônia, 14., 2009, Natal. Anais... São José dos Campos: Instituto Nacional de Pesquisas Espaciais, 2009, v. 1, p. 5359-5365.

PINTO, A.; HAYASHI, S.; AMARAL, P.; SALOMÃO, R.; SOUZA JÚNIO, C.; SAMPAIO, L. Boletim Transparência Florestal APA Triunfo do Xingu (agosto de 2010 a julho de 2011). Belém: IMAZON, 2011.

RANGEL, M. E. S. Dinâmica espacial e contingências socioambientais da hanseníase no estado do Maranhão: avaliação de riscos e vulnerabilidades em áreas hiperendêmicas. 2016. 141 f. Tese (Doutorado em Geografia Física), Universidade de São Paulo, São Paulo, 2016. Disponível em: $<$ https://teses.usp. br/teses/disponiveis/8/8135/tde-02122016-122002/pt-br.php>. Acesso em: 9 out. 2020 .

SILVESTRINI, R. A. B.; SOARES-FILHO, B.; NEPSTAD, D.; COE, M. T.; RODRIGUES, H.; ASSUNÇÃO, R. Simulating fire regimes in the Amazon in response to climate change and deforestation. Ecological Applications, v. 21, n. 5, p. 1573-1590, jul. 2011.

SOUZA JÚNIOR, C.; FONSECA, A.; NUNES, S.; SALOMÃO, R.; RIBEIRO, J.; MARTINS, H. Desmatamento em Áreas Protegidas. Belém: IMAZON, 2018. Disponível em: <http://imazon.org.br/PDFimazon/Portugues/livros/UCS\%20 mais\%20desmatadas\%20Amazonia_2012-2015.pdf>. Acesso em: 12 out. 2020. 